\title{
Anisotropic Structural Dynamics of Few-Layer Black Phosphorus Revealed by Ultrafast Electron Microscopy
}

\author{
Ye-Jin Kim ${ }^{1,2}$ and Oh-Hoon Kwon ${ }^{1,2^{*}}$ \\ 1. Department of Chemistry, School of Natural Science, Ulsan National Institute of Science and \\ Technology, Ulsan 44919, Korea \\ 2. Center for Soft and Living Matter, Institute for Basic Science, Ulsan 44919, Korea \\ * Corresponding author: ohkwon@unist.ac.kr
}

Today, we witness an avalanche of research toward fascinating two-dimensional (2D) materials for application [1], beyond naturally existing graphene, from insulating hexagonal boron nitride to semiconducting transition metal dichalcogenides (TMDs) of large band gap. The recently rediscovered black phosphorus (BP) uniquely poses interesting physical properties between those of graphene and the TMDs family. BP is a direct band gap semiconductor showing layer-number-dependent band gap [2]. Its carrier mobility positions between that of TMDs and graphene [3]. These intermediary features of BP are promising for field-effect transistors, solar cells, high-speed photodetectors, and thermoelectronics.

The unique in-plane anisotropy in the optical, electrical, thermal, and mechanical properties of BP distinguishes itself from other 2D materials as a result of its puckered orthorhombic structure: armchair (AC) structure along one axis and zigzag (ZZ) along the orthogonal one (see Fig. 1a); moreover, efficiencies in the electron and heat transport directions are orthogonal [4]. A new avenue may open to the fabrication of devices of novel functionality if we fully take advantage of the unique anisotropic properties. However, the knowledge of electronic and nuclear structural dynamics in BP upon external stimuli is requisite to this end.

In this work, we study the structural dynamics in BP (6 to 20 layers) on the timescales spanning picosecond (ps) to microsecond ( $\mu \mathrm{s}$ ) upon femtosecond (fs)/nanosecond (ns)-pulsed photoexcitation. The optical, electronic, thermal, and mechanical properties of few-layer to bulk BP have been intensively investigated in experimental and theoretical aspects in recent several years. In the nonequilibrium regime, carrier and coherent phonon in and across the layers of BP, respectively, have been studied using ultrafast optical methods [5, 6]. However, the in-plane structural dynamics have not been accessed because of the insensitivity of the optical approach and the lack of local selectivity better than the dimension of typical thin BP flakes when ultrafast diffraction methods are used.

Here, we employed ultrafast electron microscopy (UEM) to probe dynamic structural response of BP to pulsed light. The optical pump pulse excited a BP flake supported on a graphene-loaded silicon nitride and functions as a clocking pulse for the following dynamics. The brief, photoelectron packet generated from a photocathode by another pulse, synchronized to and time delayed $(\Delta t)$ with respect to the clocking pulse, took a snap shot (a micrograph or a diffractogram) of a fleeting BP structure. A series of such the timeframes provided a movie of the structural dynamics therein (Fig. 1b). The photoexcitation with a brief pump pulse resembles the practical environment of (opto)electronic devices operating under high field and/or dynamic field. In this regime, carriers are excited with non-equilibrium distributions dominating the device performances. This calls for examining the dynamic response of BP under photoexcitation, especially the evolution of electronic and structural anisotropy, through excitation- and probe-polarization-controlled UEM measurements. 
From the dynamics of local Bragg diffraction of BP flakes upon ns photoexcitation, we reveal a thermally induced non-equilibrium disordered (pre-melt) phase following electron-phonon and phononphonon interactions. The emergence of the transient phase is found to be anisotropic in the excitation polarization and in the in-plane structure (Fig. 2). The anisotropy persists until the full relaxation to the equilibrium phase. By systematically varying the polarization and fluence of the photoexcitation, the probe axis ( $\mathrm{AC}$ or $\mathrm{ZZ}$ ), and the $\mathrm{BP}$ flakes, a threshold effect for the excitation of phonon and its dependence on the in-plane anisotropic structure are also disclosed.

\section{References:}

[1] M. Xu, T. Liang, M. Shi and H. Chen, Chem. Rev. 113 (2013), p. 3766.

[2] X. Wang, et al., Nat. Nanotechnol. 10 (2015), p. 517.

[3] H. Liu, A. T. Neal, Z. Zhu, Z. Luo, X. Xu, D. Tomanek and P. D. Ye, ACS Nano 8 (2014), p. 4033.

[4] H. Liu, H. S. Choe, Y. Chen, J. Suh, C. Ko, S. Tongay and J. Wu, Appl. Phys. Lett. 111 (2017), p. 102101.

[5] X. Miao, G. Zhang, F. Wang, H. Yan and M, Ji, Nano Lett. 18 (2018), p. 3053.

[6] S. Ge, et al. Nano Lett. 15 (2015), p. 1650.

[7] The authors acknowledge funding from the Institute for Basic Science (IBS-R020-D1), Korea and the Samsung Advanced Institute of Technology (SAIT)'s Global Research Outreach (GRO) Program.
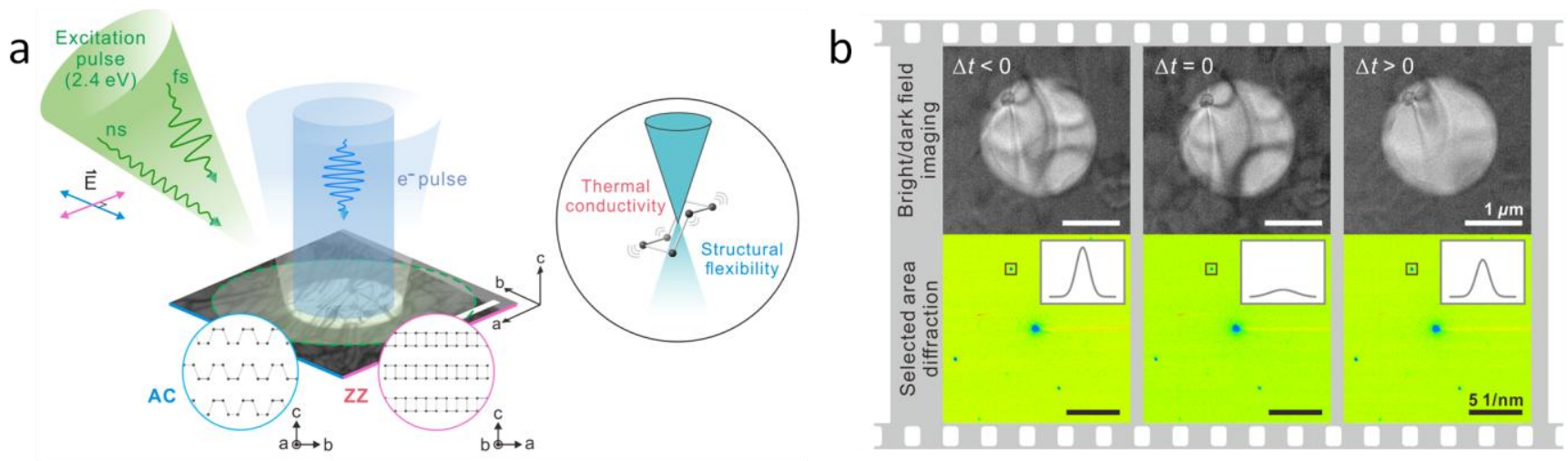

Figure 1. Probing the structural dynamics of black phosphorus using UEM. (a) Schematic setup. (b) Representative time-resolved micrographs and diffractograms upon photoexcitation.

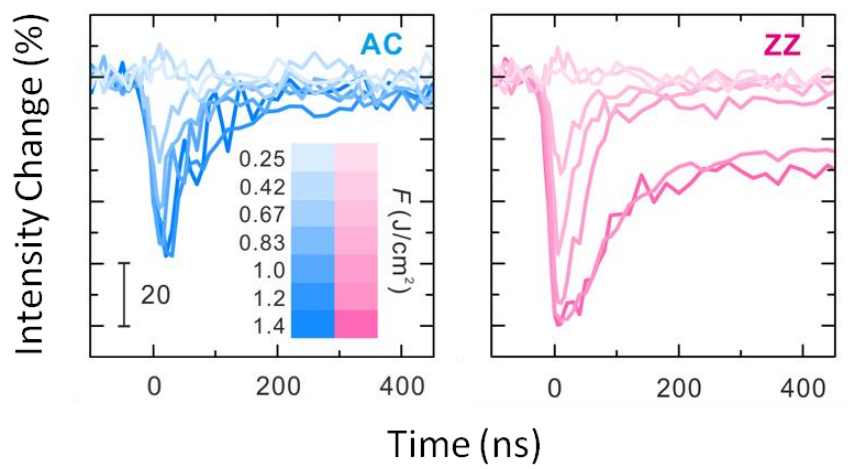

Figure 2. Fluence-dependent anisotropic response of the structural dynamics of few-layer black phosphorus along $\mathrm{AC}$ and $\mathrm{ZZ}$ directions. 